tisches Taktieren führten in Polen aber immer wieder zum Zerbrechen der fragilen Koalitionen mit personell wie programmatisch oftmals kaum zu integrierenden Parteien. Die gegenwärtige Koalition muss hier keine Ausnahme bilden. Wenn sie bisher auch nicht an Querelen zwischen den Partnern gescheitert ist, so erscheint es durchaus möglich, dass sie durch eine Unfähigkeit zu Reformen ebenfalls Gefahr läuft, das Schicksal der vergangenen Regierungsbündnisse zu teilen. Die Kohabitation mit dem stark parteilich agierenden Präsidenten könnte hierzu weiter beitragen.

Darüber hinaus ist davon auszugehen, dass die nächsten Wahlen keine dem Jahr 2007 vergleichbare Mobilisierung erleben werden. Insbesondere bei einem Scheitern der Regierung wird aller Voraussicht nach die Enttäuschung zu großer Wahlenthaltung führen, die traditionell eher der PiS nutzt, die über eine treue und wahlwillige Stammwählerschaft verfügt. Nicht umsonst riefen führende PiS-Politiker kurz vor dem Wahltermin im vergangenen Oktober beinahe unverhohlen dazu auf, den Wahlen fernzubleiben. Eine dauerhafte Veränderung wird jedoch wahrscheinlich das Verschwinden von Samoobrona und der Liga der Polnischen Familien von der politischen Bühne sein. Eventuell können Teile dieser Gruppierungen organisatorisch in der PiS aufgehen. Ihr Kernthema, der Kampf des traditionellen, ländlichen Polen gegen den modernen, vom Ausland dominierten städtischen Teil des Landes, haben die Kaczyńskis gegenwärtig fest besetzt. ${ }^{31}$ Dieses Thema wird im Verlauf der weiteren sozioökonomischen Transformation des Landes noch längere Zeit von erheblicher Bedeutung bleiben. Und so lange wird auch PiS ein bestimmender Akteur auf der politischen Bühne in Warschau sein.

In der Gesamtschau ist der Regierungswechsel in Polen insofern eher als oberflächliche Beruhigung denn als Ankerpunkt einer mittel- oder gar langfristigen Stabilisierung des politischen Gefüges zu sehen. Und er war auch nicht ein besonderer Ausweis demokratischer Reife der polnischen Bevölkerung angesichts bedenklicher Machtpraktiken der alten Führung, sondern eher das übliche Zeichen des Verdrusses über eine weitere abgewirtschaftete Regierung.

31 Den größten Teil ihrer Unterstützung zogen sie zudem aus der Angst vor den möglichen negativen Folgen des EU-Beitritts. Letztlich war zu erwarten, dass bei einem dauerhaften Ausbleiben der ausgemalten Schreckensszenarien auch das Interesse an seinen Erfindern und Pflegern über kurz oder lang abebben würde.

\title{
Die Transformation des russischen Parteiensystems: Regimestabilisierung durch personalisierte Institutionalisierung
}

\author{
Petra Stykow
}

Die Bilanz der achtjährigen Präsidentschaft Vladimir Putins (Januar 2000 bis Mai 2008) ist zwiespältig: Einerseits wurde in diesem Zeitraum mit einem anhaltenden Wirtschaftswachstum von jährlich über sechs Prozent, gewachsener politischer und sozialer Stabilität sowie der Rückkehr Russlands in die globale Arena eine einzigartige Erfolgsgeschichte geschrieben. 
Andererseits hat das hybride politische System des postkommunistischen Russlands noch deutlicher als unter Boris Jelzin (im Folgenden: El'cin) autoritäre Züge angenommen: Es weist formale Basisinstitutionen moderner Demokratien auf, aber Wahlen als Mechanismen der Elitenrekrutierung sind noch weiter ausgehöhlt worden, die horizontale und vertikale Gewaltenteilung ist unter der Übermacht der Präsidialexekutive faktisch suspendiert, die auch nicht-staatliche Akteure und Handlungssphären weithin kontrolliert. Das Parteiensystem wird von der Partei „Einiges Russland“, einer Patronagepartei des Kreml, dominiert. Ihr steht gegenwärtig keinerlei relevante Opposition gegenüber. Die Entscheidung über Sieg und Niederlage im Wettbewerb der politischen Eliten ist dem Elektorat weitgehend entzogen, dennoch schöpft das Regime Legitimität aus einer breiten Zustimmung der Bürger.

\section{Stabilisierung ohne Demokratisierung}

Ob das russische politische System heute so autoritär ist wie das der Vereinigten Arabischen Emirate, Kambodschas und Ruandas oder noch immer als eine Demokratie, wenngleich mit schwerwiegenden Defekten, anzusehen ist, wird in der international vergleichenden Forschung unterschiedlich beurteilt. ${ }^{1}$ In Fallstudien und im intraregionalen Vergleich setzt sich zunehmend die Diagnose „kompetitiver Autoritarismus“ durch. ${ }^{2}$ In einem politischen System dieses Typs werden die formal-demokratischen Spielregeln so schwerwiegend verletzt, dass es nicht mehr als Subtyp der Demokratie (zum Beispiel „defekte“ oder „illiberale Demokratie“), sondern als eine Form der Autokratie gilt. ${ }^{3}$ Über die Bedeutung von Wahlen sind sich zwar alle relevanten Akteure im System einig, aber die stärkste Akteursgruppe kontrolliert und manipuliert den politischen Wettbewerb und gegebenenfalls auch seine Ergebnisse zu ihren Gunsten. Dies geschieht insbesondere mittels informeller Praktiken. Kurz gesagt: „Competition [is] real, but unfair." 4

1 Der „Freedom House Index “ führt Russland seit 2005 mit dem Status „Not Free“ (http://www. freedomhouse.org, Abruf am 21. September 2008), der „Bertelsmann Transformation Index“ bescheinigt einen „eingeschränkten“ Stand der politischen und wirtschaftlichen Transformation (http://www.bertelsmann-transformation-index.de, Abruf am 21. September 2008), während die Demokratiequalität des politischen Systems der Putin-Ära im „Polity IV“-Projekt für geringfügig höher angesehen wird als in den 1990er Jahren (http://www.systemicpeace.org/polity/polity4. htm, Abruf am 21. September 2008).

2 Zum Beispiel Marc Morjé Howard / Philip G. Roessler, Liberalizing Electoral Outcomes in Competitive Authoritarian Regimes, in: American Journal of Political Science, 50. Jg. (2006), H. 2, S. 365 - 381; Regina Smyth / Anna Lowry / Brandon Wilkening, Engineering Victory: Institutional Reform, Informal Institutions, and the Formation of a Hegemonic Party Regime in the Russian Federation, in: Post-Soviet Affairs, 23. Jg. (2007), H. 2, S. 118 - 137; Steven Levitsky / Lucan A. Way, The Rise of Competitive Authoritarianism, in: Journal of Democracy, 13. Jg. (2002), H. 2, S. $51-65$.

3 Zur Konzeptbildung für hybride politische Systeme in der Grauzone vgl. insbesondere: David Collier / Steven Levitsky, Democracy with Adjectives: Conceptual Innovation in Comparative Research, in: World Politics, 49. Jg. (1997), H. 3, S. 430 - 451; Marc Morjé Howard / Philip G. Roessler, a.a.O. (Fn. 2); Steven Levitsky / Lucan A. Way, a.a.O. (Fn. 2).

4 Steven Levitsky / Lucan A. Way, Competitive Authoritarianism: The Emergence and Dynamics of Hybrid Regimes in the Post-Cold War Era, unveröffentlichtes Manuskript, 2006, S. 1 (http://sitemaker.umich.edu/comparative.speaker.series/files/levitsky_with_bib.pdf, Abruf am 6. Oktober 2008). 
Anders als in geschlossenen Autokratien sind Wahlsiege der Opposition in kompetitiven Autokratien zwar unwahrscheinlich, nicht aber ausgeschlossen. Die hegemonialen Eliten können sie nicht zur bloßen Farce verkommen lassen, weil sie auf die Legitimierung ihrer Herrschaft durch das Wählervotum angewiesen sind. Kompetitive Autokratien sind also inhärent instabil. Deshalb widmet sich die aktuelle Forschung der Frage, ob, wie und wem es unter welchen Umständen gelingt, solche Regime ohne Systemwechsel zu stabilisieren oder aber - etwa in Folge „bunter Revolutionen“ oder externer Demokratieförderung - zu demokratisieren.

Das politische Systems Russlands in der Putin-Ära ist ein aussagekräftiges Beispiel dafür, dass Regime des kompetitiven Autoritarismus sich endogen stabilisieren können, wenn es der regierenden Gruppe gelingt, den intra-elitären Wettbewerb um Machtressourcen einzuhegen, Kooperation zwischen rivalisierenden Gruppen zu erzwingen und die Unterstützung der Wählerschaft zu sichern. Als Mittel dafür stehen keineswegs nur informelle Praktiken in arkanpolitischen Sphären zur Verfügung, sondern auch formale Institutionen. Putins Amtszeit demonstriert, wie institutionelle Reformen durch eine hegemoniale Akteursgruppe sukzessive erarbeitet und lanciert werden können. In der Zusammenschau ergeben die einzelnen Dimensionen dieser Politik das Projekt eines ambitionierten Umbaus des politischen Systems, mit dem die politische Entscheidungsmacht konzentriert und Fortschritte in der Staatskapazität erzielt wurden. Systematische Elemente dieses Projekts waren:

(1) der Abbau der horizontalen Gewaltenteilung durch die Umwandlung der Regierung in ein Präsidialkabinett mit „technischem Premier“ und des Parlaments in eine Abstimmungsmaschine der Präsidialadministration, durch die politische Kontrolle über die Justiz und einen großen Teil der relevanten Medien, vor allem im Rundfunk;

(2) der Abbau der vertikalen Gewaltenteilung durch die Föderalismusreformen und die faktische Umwandlung des Bundes- in einen Einheitsstaat;

(3) umfassende Reformen des Ministerial-, Verwaltungs- und Justizapparats sowie Reformen des Rechts, die auf Rationalisierung und Effizienzsteigerung des Staates zielten;

(4) die Neuordnung der Beziehungen zwischen Staat und nicht-staatlichen Akteuren, das heißt zivilgesellschaftlichen Vereinigungen, Verbänden und Parteien, gekennzeichnet durch die verstärkte Regulierung des intermediären Raums, die Einrichtung konsultativer Gremien bei staatlichen Agenturen und die gezielte Patronage präsidententreuer Organisationen;

(5) die Formulierung einer Legitimationsideologie in Gestalt des Konzepts der „gelenkten" beziehungsweise „souveränen Demokratie“, die sowohl innen- als auch außenpolitische Funktionen erfüllt. ${ }^{5}$

In der öffentlichen Wahrnehmung gelten nicht nur die informellen Praktiken und Institutionen des so genannten Systems Putin als autoritär. Vielmehr werden gerade die Reformen der formalen Institutionen als deutlicher Nachweis des Abgleitens Russlands in den Autoritarismus interpretiert. Die tatsächliche Situation ist komplizierter, wie im Folgenden anhand der Transformation des Parteiensystems gezeigt werden soll, das einen tiefgreifen-

5 Ausführlicher über diese Dimensionen des Putinschen Reformprojekts Michael McFaul / Kathryn Stoner-Weiss, The Myth of the Authoritarian Model. How Putin's Crackdown Holds Russia Back, in: Foreign Affairs, 87. Jg. (2008), H. 1, S. 68 - 84; Petra Stykow, Die autoritäre Konsolidierung des politischen Systems in der Ära Putin, in: Hans-Henning Schröder / Heiko Pleines (Hrsg.), Länderbericht Russland, Bonn i.E.; Regina Smyth / Anna Lowry / Brandon Wilkening, a.a.O. (Fn. 2), S. $122 \mathrm{ff}$. 
den Wandel erfahren hat. Dabei sind die formal-institutionellen Reformen meist nicht per se als undemokratisch zu bewerten. Sie führen jedoch aufgrund der Kontextbedingungen, unter denen sie wirken, zu undemokratischen Effekten, insbesondere zur faktischen Strangulierung des politischen Wettbewerbs.

\section{Das unterinstitutionalisierte Parteiensystem der 1990er Jahre}

\subsection{Befund}

Das russische Parteiensystem der 1990er Jahre war dynamisch, zentrifugal und dadurch geprägt, dass jegliche Anzeichen einer Institutionalisierung - im Sinne eines „process by which organizations and procedures acquire value and stability " 6 - ausblieben. In der umfangreichen Forschungsliteratur zu diesem Problem werden folgende Merkmale des Parteiensystems herausgearbeitet ${ }^{7}$ :

(1) Fast alle Parteien waren kurzlebige Gebilde. Von den 13 Parteien, die 1993 um Wählerstimmen konkurriert hatten, waren bis 1999 bereits acht wieder von der politischen Bildfläche verschwunden, und nur acht der 43 Parteien, die zu den Duma-Wahlen von 1995 angetreten waren, taten dies auch 1999 wieder. Die einzigen Parteien, die über das gesamte Jahrzehnt beständig am Wettbewerb um Wähler teilnahmen, waren die Kommunistische Partei der Russischen Föderation (KPRF), die Liberal-Demokratische Partei (LDPR) und die sozialliberale Partei „Jabloko“. Dennoch wurde deren Persistenz nur vereinzelt als Hinweis auf eine beginnende Konsolidierung eines Mehrparteiensystems interpretiert. Mit Ausnahme der KPRF handelte es sich bei allen Parteien um mitgliederschwache, hoch personalisierte Organisationen, deren Bestandsaussichten ausschließlich von ihrem Führungspersonal abhingen.

6 Samuel Huntington, Political Order in Changing Societies, New Haven 1968, S. 12; zu Kriterien der Institutionalisierung vgl. Scott Mainwaring, Party Systems in the Third Wave, in: Journal of Democracy, 9. Jg. (1998), H. 3, S. 67 - 81; Richard Rose / Neil Munro / Stephen White, Voting in a Floating Party System: The 1999 Duma Election, in: Europe-Asia Studies, 53. Jg. (2001), H. 3 , S. 419 - 443, S. $420 \mathrm{ff}$.

7 Die folgenden Befunde sind, wenn nicht anders vermerkt, zusammengetragen aus: Jack Bielasiak, The Institutionalization of Electoral and Party Systems in Postcommunist States, in: Comparative Politics, 34. Jg. (2002), H. 2, S. 189 - 210; M. Steven Fish, The Advent of Multipartism in Russia, in: Post-Soviet Affairs, 11. Jg. (1995), H. 4, S. 340 - 383; Vladimir Gelman, From „Feckless Pluralism“ to „Dominant Power Politics“? The Transformation of Russia’s Party System, in: Democratization, 13. Jg. (2006), H. 4, S. 545 - 561; Robert G. Moser, The Consequences of Russia's Mixed-Member Electoral System, in: Matthew Soberg Shugart / Martin P. Wattenberg (Hrsg.), Mixed-Member Electoral Systems: The Best of Both Worlds? Oxford 2001, S. 494 - 518; William M. Reisinger / Arthur H. Miller / Vicky L. Hesli, Ideological Divisions and Party-building Prospects in Post-Soviet Russia, in: Matthew Wyman / Stephen White / Sarah Oates (Hrsg.), Elections and Voters in Post-communist Russia, Cheltenham 1998, S. 136 - 166; Richard Rose / Neil Munro / Stephen White, a.a.O. (Fn. 6); Regina Smyth, Building State Capacity from the Inside Out: Parties of Power and the Success of the President's Reform Agenda in Russia, in: Politics \& Society, 30. Jg. (2002), H. 4, S. 555 - 578; Kathryn Stoner-Weiss, The Limited Reach of Russia's Party System: Underinstitutionalization in Dual Transitions, in: Politics \& Society, 29. Jg. (2001), H. 3, S. 385 - 414; Stephen White / Richard Rose / Ian McAllister, How Russia Votes. New York 1997. 
(2) Die Parteistrukturen waren nur rudimentär entwickelt und gingen kaum über den zentralen Organisationsapparat hinaus. In der Provinz traten Politiker selten als Repräsentanten von Parteien auf, was sich bei Wahlen zu den regionalen Parlamenten und in ihrer Tätigkeit deutlich zeigte. ${ }^{8}$ Auch erwies sich keine einzige der nationalen Parteien als fähig, bei den Wahlen zur Staatsduma in allen territorialen Einerwahlkreisen eigene Kandidaten zu nominieren. Weil seit 1993 ein Grabenwahlsystem galt, das Elemente des Verhältnisund des Mehrheitswahlrechts ohne Verrechnung der Mandate kombinierte ${ }^{9}$, ergab es sich daher, dass Duma-Abgeordnete, die in den Regionen Direktmandate erzielten, nur selten Parteien angehörten. So erhielten noch 1999 vier Parteien 54 Prozent der Listenplätze im Proporz-Segment, zusammen aber nur acht Prozent der Direktmandate im Majorz-Segment. In 51 Prozent der Einerwahlkreise siegten hingegen Kandidaten ohne jegliche Parteibindung (vgl. Tabelle 1).

(3) Das Parteiensystem war hoch fragmentiert und ideologisch polarisiert (vgl. Tabelle 2), was sich in der starken parlamentarischen Präsenz der Kommunisten widerspiegelte. Diese waren allerdings - zusammen mit kleinen wirtschaftsliberalen Parteien - die einzigen Organisationen mit einem klaren politischen Profil. Die ideologische Zuordnung zu Parteienfamilien und die Bildung von Allianzen folgten nur partiell den in westlichen Demokratien geläufigen Mustern. Dies zeigte sich beispielsweise anhand der Liberal-Demokraten, deren Führer Vladimir Žirinovskij nationalistische bis rechtsradikale Positionen vertrat, aber auch anhand höchst eigentümlicher „rot-brauner“ Allianzen von Kommunisten und extremen Nationalisten an den äußeren Rändern des politischen Spektrums. Aufgrund dieser Besonderheit halten einige Autoren die gängige Zuordnung des Parteiensystems zu Sartoris Typ des ideologisch „polarisierten Pluralismus “ für nicht zutreffend. ${ }^{10}$ Tendenziell nahm die Polarisierung des Parteiensystems gegen Ende der 1990er Jahre übrigens deutlich ab. In entsprechenden Untersuchungen wird sogar von einem Trend zur Konvergenz der Parteiprogramme gesprochen. ${ }^{11}$

(4) Die große Zahl parteiunabhängiger Kandidaten, die geringe Loyalität von Mandatsträgern gegenüber ihrer Partei und die besonderen Anreize der parlamentarischen Arena führten dazu, dass auch die Fraktionen in der Duma instabil waren. Die erheblichen Wanderungen zwischen den Fraktionen, die ein großer Teil der Abgeordneten sofort nach der Wahl und im weiteren Verlauf der Wahlperiode unternahm, sind selbst Indikatoren einer Parteienbildung im Fluss. Sie veränderten mitunter sogar die Mehrheitsverhältnisse in der Duma (vgl. Tabelle 3). Analytisch muss also das Parteiensystem, dessen Elemente am Wettbewerb um Wählerstimmen teilnahmen (electoral party system), von einem mit ihm interagierenden Parteiensystem unterschieden werden, dessen Fluidität sich zwischen den Wahlen in der parlamentarischen Arena entfaltete (legislative party system). ${ }^{12}$

8 Kathryn Stoner-Weiss, a.a.O. (Fn. 7).

9 Zum russischen Wahlsystem vgl. Dieter Nohlen, Wahlrecht und Parteiensystem. Zur Theorie und Empirie der Wahlsysteme, 5. Auflage, Opladen 2007, Kap. 8.8; Robert G. Moser, a.a.O. (Fn. 7).

10 Giovanni Sartori, Parties and Party Systems: A Framework for Analysis, Cambridge 1976, S. 119 - 129; zur Einordnung des russischen Parteiensystems vgl. Regina Smyth, a.a.O. (Fn. 7) vs. $M$. Steven Fish, a.a.O. (Fn. 7); Robert G. Moser, Unexpected Outcomes: Electoral Systems, Political Parties, and Representation in Russia, Pittsburgh 2001, S. 128 - 131.

11 Vladimir Gelman, a.a.O. (Fn. 7), S. 554.

12 Ähnliche Phänomene werden auch in anderen instabilen Parteiensystemen beobachtet, zum Beispiel in Italien, vgl. Daniela Giannetti / Michael Laver, Party System Dynamics and the Making 


\begin{tabular}{|l|c|c|c|c|c|c|}
\hline Tabelle 1: Parlamentswahlen in Russland (1993 bis 2008) \\
\hline Wahlen* & 12.12 .1993 & 17.12 .1995 & 19.12 .1999 & 7.12 .2003 & 2.12 .2007 \\
\hline Wahlbeteiligung & $54,3 \%$ & $64,4 \%$ & $61,7 \%$ & $55,7 \%$ & $63,7 \%$ \\
\hline $\begin{array}{l}\text { Anzahl der Parteien / Bündnisse, die } \\
\text { sich im Proporz-Segment zur Wahl } \\
\text { stellten }\end{array}$ & 13 & 43 & 26 & 33 & 11 \\
\hline $\begin{array}{l}\text { Anzahl der Parteien / Bündnisse, } \\
\text { welche die Sperrklausel überwanden }\end{array}$ & 8 & 4 & 6 & 4 & 4 \\
\hline $\begin{array}{l}\text { Anzahl der Parteien, die im Majorz- } \\
\text { Segment Direktmandate erzielten }\end{array}$ & 12 & 23 & 13 & 11 & - \\
\hline $\begin{array}{l}\text { Anteil der Stimmen für unabhängige } \\
\text { Kandidaten im Majorz-Segment }\end{array}$ & $45,2 \%$ & $31,2 \%$ & $41,7 \%$ & $26,8 \%$ & - \\
\hline $\begin{array}{l}\text { Gesamtzahl der Parteien im Parlament } \\
\text { (Mandate in beiden Segmenten) }\end{array}$ & 12 & 23 & 14 & 12 & 4 \\
\hline $\begin{array}{l}\text { * Grabenwahlsystem 1993, 1995, 1999, 2003; Verhältniswahlsystem 2007. } \\
\text { Quelle: Angaben der Zentralen Wahlkommission der Russischen Föderation (http://www.izbirkom.ru, } \\
\text { Abruf am 10. September 2008) für 2003 und 2007 beziehungsweise zitiert nach der Datenbank, Politi- } \\
\text { cal Transformation and the Electoral Process in Post-Communist Europe“ an der Universität Essex } \\
\text { (http://www.essex.ac.uk/elections, Abruf am 10. September 2008) für 1993, 1995, 1999. }\end{array}$ \\
\hline
\end{tabular}

(5) Die Wählervolatilität, die in allen postkommunistischen Parteiensystemen vergleichsweise groß war, erreichte in Russland außerordentlich hohe Werte (vgl. Tabelle 2). Ihr Ausmaß bleibt selbst dann noch beachtlich, wenn man die Kurzlebigkeit der einzelnen Parteien in Rechnung stellt und Wählerwanderungen nur zwischen Parteienfamilien misst. ${ }^{13}$ Dennoch begannen sich im Laufe des Jahrzehnts Parteiidentifikationen zu entwickeln; die Wähler lernten, Verbindungen zwischen ihren Erfahrungen mit dem politischen und wirtschaftlichen Wandel und den Positionen von Politikern und Parteien herzustellen. ${ }^{14}$ Insgesamt waren die Einstellungen der Bürger gegenüber Wahlen und Parteien überaus ambivalent. Eine Mehrheit hielt sie für notwendige politische Institutionen, vertraute ihnen aber nicht. Die Wahlbeteiligung war relativ niedrig, wenngleich in weniger dramatischem Ausmaß, als mitunter behauptet wird (vgl. Tabelle 1). ${ }^{15}$

and Breaking of Italian Governments, in: Electoral Studies, 20. Jg. (2001), H. 4, S. 529 - 553; Michael Laver / Kenneth Benoit, The Evolution of Party Systems between Elections, in: American Journal of Political Science, 47. Jg. (2003), H. 2, S. $215-233$.

13 Jack Bielasiak, a.a.O. (Fn. 7), S. 198 - 200; Vladimir Gelman, a.a.O. (Fn. 7), S. 547.

$14 \mathrm{Zu}$ kontroversen Befunden und Interpretationen vgl. Ted Brader / Joshua A. Tucker, The Emergence of Mass Partisanship in Russia, 1993-1996, in: American Journal of Political Science, 45. Jg. (2001), H. 1, S. 69 - 83; Timothy Colton, Transitional Citizens: Voters and Elections in PostSoviet Russia, Cambridge 2000, S. 150; Arthur H. Miller / Gwyn Erb / William M. Reisinger / Vicki L. Hesli, Emerging Party Systems in Post-Soviet Societies: Fact or Fiction, in: Journal of Politics, 62. Jg. (2000), H. 2, S. 455 - 490; Mikhail Myagkov / Peter C. Ordeshook, The Trail of Votes in Russia's 1999 Duma and 2000 Presidential Elections, in: Communist and Post-Communist Studies, 34. Jg. (2001), H. 3, S. 353 - 370; Robert G. Moser, a.a.O. (Fn. 10), Kap. 7; Stephen White / Richard Rose / Ian McAllister, a.a.O. (Fn. 7); Stephen Whitefield / Geoffrey Evans, Class, Markets and Partisanship in Post-Soviet Russia: 1993-96, in: Electoral Studies, 18. Jg. (1999), H. 2, S. $155-178$.

15 Timothy J. Colton / Michael McFaul, Are Russians Undemocratic? Carnegie Endowment for International Peace, Washington, DC 2001, S. 12 f.; William Mishler / Richard Rose, Trust, Distrust and Skepticism, in: Journal of Politics, 59. Jg. (1997), H. 2, S. $418-451$. 


\begin{tabular}{|c|c|c|c|c|c|c|}
\hline \multirow[b]{2}{*}{ Indikator } & \multicolumn{2}{|c|}{ effektive Zahl der Parteien } & \multirow{2}{*}{$\begin{array}{l}\text { verschenkte } \\
\text { Stimmen }{ }^{\mathrm{c}}\end{array}$} & \multirow{2}{*}{$\begin{array}{l}\text { Offenheit } \\
\text { des Parteien- } \\
\text { systems }^{\mathrm{d}}\end{array}$} & \multirow{2}{*}{$\begin{array}{c}\text { ideologische } \\
\text { Polarisierung }\end{array}$} & \multirow{2}{*}{$\begin{array}{l}\text { elektorale } \\
\text { Volatilität }\end{array}$} \\
\hline & $\begin{array}{c}\text { auf } \\
\text { Wählerebene }\end{array}$ & $\begin{array}{c}\text { im } \\
\text { Parlament }\end{array}$ & & & & \\
\hline $1993-95$ & 7,6 & 8,5 & $16,4 \%$ & - & $10,7 \%$ & - \\
\hline $1995-99$ & 10,7 & 5,7 & $38,3 \%$ & $17,9 \%$ & $34,9 \%$ & $42,5 \%$ \\
\hline 1999 & 6,8 & & $13,3 \%$ & $32,9 \%$ & $25,1 \%$ & $52,1 \%$ \\
\hline $2000-01^{*}$ & - & 7,8 & - & - & - & - \\
\hline $2001-03$ & - & 4,7 & - & - & - & - \\
\hline $2003-07$ & 5,3 & 2,0 & $22,9 \%$ & $13,1 \%$ & $11,6 \%$ & $19,7 \%$ \\
\hline $2007-$ & 2,3 & 1,9 & $7,2 \%$ & $\left(8,4 \%{ }^{* *}\right)$ & $12,7 \%$ & $26,9 \%$ \\
\hline
\end{tabular}

${ }^{a}$ ENEP (effective number of parties at the electoral level) nach Markku Laakso / Rein Taagepera, berechnet nach Stimmanteilen (bis 2003 nur im Proporz-Segment), Quelle: Vladimir Gel'man, a.a.O. (Fn. 41), S. 914.

${ }^{\mathrm{b}}$ ENPP (effective number of parties at the parliamentary level) nach Laakso / Taagepera, berechnet nach Mandatsanteilen, Quelle: Vladimir Gel'man, a.a.O. (Fn. 41), S. 914.

${ }^{\mathrm{c}}$ Anteil der Stimmen, die für Parteien abgegeben wurden, die an der Sperrklausel scheiterten (ohne "Gegen-Alle“-Stimmen), eigene Berechnungen nach Angaben der Zentralen Wahlkommission.

d Anteil neuer Parteien an allen Duma-Mandaten, eigene Berechnungen nach Angaben der Zentralen Wahlkommission.

${ }^{\mathrm{e}}$ Mandatsanteil der KPRF nach Angaben der Zentralen Wahlkommission; Indikator der Polarisierung nach Frye: Anteil der Kommunisten (Antikommunisten) bei Dominanz von Antikommunisten (Kommunisten) in der Exekutive; die Polarisierung gilt als hoch, wenn der Wert mindestens 20 Prozent beträgt, vgl. Timothy Frye, The Perils of Polarization: Economic Performance in the Postcommunist World, in: World Politics, 54. Jg. (2002), H. 3, S. 308 - 337, S. $312-318$.

${ }^{\mathrm{f}}$ Summe aller Zugewinne von Parteien gegenüber den vorangegangenen Wahlen (Pedersen-Index); Quelle: Jack Bielasiak, a.a.O. (Fn. 7), S. 201, für 1995 und 1999, eigene Berechnungen anhand der Wahlergebnisse für 2003 und 2007.

* Die Wahlperiode 2000 bis 2003 wurde wegen der Reorganisation der Duma-Parteien im Frühjahr 2001 in zwei Teilperioden zerlegt, siehe Abschnitt 3.2.

** Anteil der Partei „Gerechtes Russland“, die aus dem Zusammenschluss dreier Parteien entstand; eine von ihnen war allerdings mit 8,2 Prozent der Mandate auch in der vorangegangenen Duma vertreten.

Zusammenfassend wird das russische Parteiensystem der 1990er Jahre in der Literatur als ein - typischer oder extremer ${ }^{16}$ - Fall zäher Unterinstitutionalisierung beschrieben, wie sie für postkommunistische Demokratien und darüber hinaus für die meisten jungen Demokratien der „Dritten Welle“ seit 1974 charakteristisch ist. Sie signalisiert ein strukturelles Ungleichgewicht zwischen dem von Wahl zu Wahl signifikant veränderten Parteienangebot und der Wählernachfrage. In allen neuen Demokratien bleiben Muster und Regeln des Parteienwettbewerbs lange instabil; den Parteien gelingt es nicht, sich in der Gesellschaft zu verwurzeln, sie stellen eher klientelistische Patronage- beziehungsweise Führerparteien dar und genießen nur geringe Legitimität. ${ }^{17}$

16 Während Richard Rose / Neil Munro, Elections and Parties in New European Democracies, Washington, DC 2003, das russische Parteiensystem in ersterem Sinne charakterisieren, spricht Robert G. Moser, a.a.O. (Fn. 7), S. 497, von einem „extreme case of a weakly institutionalized party system".

17 Vgl. zum Beispiel Jack Bielasiak, Party Competition in Emerging Democracies: Representation and Effectiveness in Post-Communism and Beyond, in: Democratization, 12. Jg. (2005), H. 3 , 


\begin{tabular}{|c|c|c|c|c|c|}
\hline Wahlperiode & $1994-1995$ & $1996-1999$ & $2000-2003$ & $2004-2007$ & $2008-2011$ \\
\hline \multicolumn{6}{|c|}{ Anzahl der Fraktionen und Gruppen } \\
\hline konstituierende Sitzung & 9 & 7 & 9 & 4 & 4 \\
\hline Ende der Wahlperiode & 11 & 7 & 6 & 5 & \\
\hline \multicolumn{6}{|c|}{ stärkste Fraktion (Zahl der Mandate / Anteil der Mandate in Prozent) } \\
\hline laut Wahlergebnis & $\begin{array}{l}\text { Russlands } \\
\text { Wahl } \\
(70 / 15,6)\end{array}$ & $\begin{array}{l}\text { KPRF } \\
(157 / 34,9)\end{array}$ & $\begin{array}{l}\text { KPRF } \\
(113 / 25,1)\end{array}$ & $\begin{array}{l}\text { ER } \\
(222 / 49,3)\end{array}$ & $\begin{array}{l}\text { ER } \\
(315 / 70)\end{array}$ \\
\hline Ende der Wahlperiode & $\begin{array}{l}\text { LDPR } \\
(55 / 12,5) \\
\end{array}$ & $\begin{array}{l}\text { KPRF } \\
(127 / 28,9) \\
\end{array}$ & \begin{tabular}{|l|} 
ER \\
$(235 / 53,4)$ \\
\end{tabular} & $\begin{array}{l}\text { ER } \\
(303 / 68,2) \\
\end{array}$ & \\
\hline \multicolumn{6}{|l|}{ Fraktionswechsler } \\
\hline $\begin{array}{l}\text { zwischen Wahltag und } \\
\text { konstituierender Sitzung }\end{array}$ & \begin{tabular}{l|l}
106 \\
(Januar 1994)
\end{tabular} & $\begin{array}{l}105 \\
\text { (Januar 1996) }\end{array}$ & \begin{tabular}{|l|}
147 (Januar \\
$2000)$ \\
\end{tabular} & $\begin{array}{l}83 \text { (Ende } \\
\text { Januar 2004) }\end{array}$ & - \\
\hline $\begin{array}{l}\text { zwischen Wahltag und } \\
\text { Ende der Wahlperiode* }\end{array}$ & $\begin{array}{l}195 \text { (Oktober } \\
1995)\end{array}$ & $\begin{array}{l}124 \text { (Oktober } \\
1999)\end{array}$ & $\begin{array}{l}174 \\
\text { (Juli 2003) }\end{array}$ & $\begin{array}{l}99 \text { (Oktober } \\
2007)\end{array}$ & - \\
\hline $\begin{array}{l}\text { unabhängige Kandidaten } \\
\text { laut Wahlergebnis - zur } \\
\text { konstituierenden Sitzung } \\
\text { - am Ende der Wahl- } \\
\text { periode }\end{array}$ & $146-47-57$ & $77-25-32$ & $105-17-19$ & $68-17-24$ & - \\
\hline \multicolumn{6}{|c|}{$\begin{array}{l}\text { * Es handelt sich um den minimalen Wert für die Ein- und Übertritte von Abgeordneten in Parlaments- } \\
\text { fraktionen, da - mangels Daten - nur die Wanderungen zu zwei Zeitpunkten (Addition der absoluten } \\
\text { Werte) erfasst wurden, diese sich aber über die gesamte Wahlperiode mit einem typischen Höhepunkt in } \\
\text { der Mitte erstrecken; vgl. Carol Mershon / Olga Shvetsova, Parliamentary Cycles and Party Switching in } \\
\text { Legislatures, in: Comparative Political Studies, 41. Jg. (2008), H. 1, S. } 99 \text { - 127. } \\
\text { Quelle: Eigene Berechnungen nach Angaben über die Mandatsverteilung in: Stephen White / Richard Rose } \\
\text { / Ian McAllister, a.a.O. (Fn. 7), S. 184; Centre for the Study of Public Policy, University of Aberdeen / } \\
\text { The Levada Center Moscow, Russia Votes (http://www.russiavotes.org, Abruf am 12. September 2008); } \\
\text { Russlandanalysen 13/2004, S. } 6 \text { (http://www.laender-analysen.de/russland, Abruf am 12. September } \\
\text { 2008). }\end{array}$} \\
\hline
\end{tabular}

\subsection{Erklärungen}

Die Unterinstitutionalisierung von Parteiensystemen in jungen Demokratien, darunter im Russland der 1990er Jahre, ist Gegenstand einer differenzierten und fruchtbaren Diskussion. Die dabei entwickelten Erklärungen schließen an unterschiedliche Forschungstraditionen an: Strukturalistische Interpretationen argumentieren mit der Schwäche oder Instabilität historisch verwurzelter gesellschaftlicher Konfliktlinien (Cleavages) und mit den zunächst nicht vorhandenen Klassenstrukturen. Institutionalistische Erklärungen, die an elaborierte Ergebnisse der international vergleichenden Wahlsystemforschung anknüpfen, arbeiten die prägenden Wirkungen der neuen Wahl- oder Regierungssysteme auf die Gestalt der entstehenden Parteiensysteme heraus. Komplexere Modelle berücksichtigen einerseits das Zusammenspiel zwischen dem Wahlsystem und anderen politischen Institutionen,

S. 331 - 356; Scott Mainwaring / Edurne Zoco, Political Sequences and the Stabilization of Interparty Competition, in: Party Politics, 13. Jg. (2007), H. 2, S. 155 - 178; Richard Rose / Neil Munro, a.a.O. (Fn. 16). 
andererseits das je unterschiedliche institutionelle Erbe des Staatssozialismus. In allen diesen Dimensionen sind in den vergangenen Jahren auch für den russischen Fall plausible Argumentationen entwickelt worden, von denen die wichtigsten kurz skizziert seien. ${ }^{18}$

Das Argument fehlender oder instabiler Konfliktstrukturen und politischer Spaltungen hat sich als eher schwach erwiesen, da empirische Untersuchungen erstens gegenläufige Belege erbracht haben und zweitens keine direkte Kausalkette zwischen Cleavages und Wählerverhalten oder gar Institutionalisierungschancen von Parteien besteht. ${ }^{19}$ Auch der simple Bezug auf institutionelle oder politisch-kulturelle Hinterlassenschaften der sowjetischen und vorsowjetischen Zeit erklärt wenig, da Russland sich in dieser Hinsicht zwar stark von den ostmitteleuropäischen jungen Demokratien, aber wenig von der Ukraine und Belarus unterscheidet. Dennoch weisen die Parteiensysteme in diesen drei Ländern erhebliche Varianz auf. ${ }^{20}$

Vor diesem Hintergrund ist in der Forschung den „early institutional choices“ der frühen 1990 er Jahre eine besondere und zudem kumulative Bedeutung zugemessen worden. Verfassungsdesignern und Parteistrategen, eventuell unterstützt durch ausländische Berater, boten sich damals günstige Chancen „[to] engineer a party system into being without being overconcerned about economic and social preconditions “21. Diese frühen Gestaltungsentscheidungen wurden in den meisten postkommunistischen Ländern in einer politisch höchst aufgeladenen Atmosphäre getroffen, die durch unterschiedliche Akteurskonstellationen, aber auch durch je spezifische institutionelle Hinterlassenschaften des Staatssozialismus und deutlich erkennbare Übergangspfade zur Demokratie bedingt war. So dominierten in Russland zum Zeitpunkt der Verfassunggebung 1993 zwar klar die Reformer um Boris El'cin, unter den Bedingungen von Unsicherheit entschieden sie sich aber nicht für machtmaximierende Institutionenentwürfe, sondern für Mischsysteme, um einer extremen Nutzenkonzentration bei einer einzelnen Akteursgruppe entgegenzuwirken. ${ }^{22}$ Sie kombinierten dabei unterschiedliche Logiken und Prinzipien. Dies ging zu Lasten der Konsistenz der jeweiligen Institutionen und brachte ihnen das Attribut „poorly designed“ ein. Institutionelle Entscheidungen, die diesem Kalkül folgten, waren beispielsweise das semi-präsidentielle Regierungssystem, das Grabenwahlsystem mit seiner Parallelität aus Verhältnis- und relativer Mehr-

18 Für einen Überblick vgl. Henry E. Hale, Why Not Parties?, in: Comparative Politics, 37. Jg. (2005), H. 2, S. 147 - 166, S. 150 ff.; M. Steven Fish, The Impact of the 1999-2000 Parliamentary and Presidential Elections on Political Party Development, in: Vicki L. Hesli / William M. Reisinger (Hrsg.), The 1999-2000 Elections in Russia: Their Impact and Legacy, Cambridge 2003, S. $186-212$, S. $194-210$.

19 Stephen Whitefield, Political Cleavages and Post-Communist Politics, in: Annual Review of Political Science, 5. Jg. (2002), S. $181-200$.

20 Frank C. Thames, Discipline and Party Institutionalization in Post-Soviet Legislatures, in: Party Politics, 13. Jg. (2007), H. 4, S. 456 - 477; Vladimir Gelman, a.a.O. (Fn. 7), S. 549 ff.

21 Thomas F. Remington, Political Conflict and Institutional Design: Paths of Party Development in Russia, in: Journal of Communist Studies and Transition Politics, 14. Jg. (1998), H. 1, S. 201 -223, S. 202.

22 Timothy Frye, A Politics of Institutional Choice: Post-Communist Presidencies, in: Comparative Political Studies, 30. Jg. (1997), H. 5, S. 523 - 552; Michael McFaul, Institutional Design, Uncertainty, and Path Dependency during Transitions: Cases from Russia, in: Constitutional Political Economy, 10. Jg. (1999), S. 27 - 52; Robert G. Moser / Frank C. Thames, Compromise Amidst Political Conflict: The Origins of Russia's Mixed-Member System, in: Matthew Soberg Shugart / Martin P. Wattenberg (Hrsg.), a.a.O. (Fn. 7), S. 255 - 275. 
heitswahl sowie der asymmetrische Vertragsföderalismus. Im Rückblick wird übrigens deutlich, dass die Reformen der letzten Jahre in vielem darauf gerichtet waren, die Ambivalenz dieser Institutionen zu korrigieren, indem formale Elemente eingebaut wurden, die auf größere Eindeutigkeit zielten und damit auch mehr typologische Klarheit erzeugten.

Die 1993 installierten Institutionen schufen in der Folgezeit Anreize für das Verhalten politischer Akteure, die weder den Bedeutungsgewinn von Parteien noch die Konzentration des Parteiensystems beförderten. Beobachter waren zunächst überrascht, dass das Mehrheitswahl-Segment des Wahlrechts keineswegs konzentrierend auf das Parteiensystem wirkte, während die Verhältniswahl nach Parteilisten die Zahl der relevanten Parteien reduzierte. Die Erklärung dafür fand sich jedoch bald in der zirkulären Interaktion von Wahlrecht und Parteiensystem, das heißt in der Kontextualisierung institutioneller Wirkungen: Das unterinstitutionalisierte, personalisierte Parteiensystem Russlands schwächte die konzentrierende Wirkung der Majorz-Regel ab. ${ }^{23} \mathrm{Ob}$ das Wahlrecht reformiert werden sollte und wenn ja, in welche Richtung, blieb in der Literatur gleichwohl umstritten. ${ }^{24}$

Die zweite Institution, der Schlüsselbedeutung für die Entwicklung des Parteiensystems beigemessen wurde, war das Regierungssystem. Seine semi-präsidentielle Ausgestaltung setzte vergleichsweise schwache Anreize für die Institutionalisierung von Parteien: Der (sich überparteilich verstehende) Präsident dominierte das Regierungssystem, Präsidentschaftswahlen wurden als Entscheidungen zwischen Personen statt im Parteienwettbewerb ausgetragen, und die Zusammensetzung des Kabinetts hing nicht mit dem Ergebnis von Parlamentswahlen zusammen. Dennoch werden in der einschlägigen Debatte nicht primär den formal-konstitutionellen Eigenschaften des Regierungssystems negative Wirkungen auf das Parteiensystem zugeschrieben, sondern meist seiner informellen Funktionsweise, die als „Superpräsidentialismus“ bezeichnet wird. ${ }^{25}$ Sie ist durch die extreme Personalisierung des Präsidentenamtes und Klientelismus bei der Herrschaftsorganisation und -ausübung ge-

23 Vgl. John T. Ishiyama / Ryan Kennedy, Superpresidentialism and Political Party Development in Russia, Ukraine, Armenia and Kyrgyzstan, in: Europe-Asia Studies, 53. Jg. (2001), H. 8, S. 1177 - 1191; Dieter Nohlen, a.a.O. (Fn. 9), Kap. 8.8; Florian Grotz, Die Entwicklung kompetitiver Wahlsysteme in Mittel- und Osteuropa: Post-sozialistische Entstehungsbedingungen und fallspezifische Reformkontexte, in: Österreichische Zeitschrift für Politikwissenschaft, 34. Jg. (2005), H. 1, S. 27 - 42; Robert G. Moser, a.a.O. (Fn. 7); Stephen White, Russia: The Authoritarian Adaptation of an Electoral System, in: Michael Gallagher / Paul Mitchell (Hrsg.), The Politics of Electoral Systems, Oxford 2005, S. 313 - 330.

24 Timm Beichelt, Demokratische Konsolidierung im postsozialistischen Europa, Opladen 2001, S. 242 - 258; Robert G. Moser, a.a.O. (Fn. 7), S. 516 ff.; Jonathan W. Riggs / Peter J. Schraeder, Russia’s Political Party System as an Impediment to Democratization, in: Demokratizatsiya, 12. Jg. (2004), H. 2, S. 265 - 293, S. 289; Neil Robinson, The Politics of Russia’s Partial Democracy, in: Political Studies Review, 1. Jg. (2003), S. 149 - 166, S. 153 f.

25 Vgl. Mikhail Myagkov / Peter C. Ordeshook, a.a.O. (Fn. 14); Terry Clark / Jill N. Wittrock, Presidentialism and the Effect of Electoral Law in Postcommunist Systems: Regime Type Matters, in: Comparative Political Studies, 38. Jg. (2005), H. 2, S. 171 - 188, und Michael McFaul, Party Formation and Non-Formation in Russia, Carnegie Endowment for International Peace, Washington, DC 2000, Working Papers, Russian and Eurasian Program, S. 12, gehören zu den seltenen Beispielen, in denen der Superpräsidentialismus vorrangig als besonders starke formale Ausprägung eines semi-präsidentiellen Regierungssystems angesehen wird; sie schreiben ihm hemmende Wirkungen für die Parteienbildung zu. Anders John T. Ishiyama / Ryan Kennedy, a.a.O. (Fn. 23), die ihm keine signifikante Wirkung attestieren. Zum Verständnis des Begriffs "Superpräsidentialismus" vgl. Neil Robinson, a.a.O. (Fn. 24). 
kennzeichnet. Konzeptionell handelt es sich dabei also nicht um eine Erweiterung der klassischen Regierungssystemtypologie, sondern um den neopatrimonialen Subtyp eines „Regimes“ im Sinne einer Anordnung von Prozeduren, die den Zugang zu den Machtressourcen eines Staates organisieren und regulieren. Zur Vermeidung von Missverständnissen wird deshalb im Folgenden der Begriff des „patronalen Präsidentialismus“ verwendet, den Henry Hale anhand postsowjetischer Beispiele in die Diskussion eingeführt hat. ${ }^{26}$

Da für diesen Regimetyp insbesondere ererbte informelle Praktiken mit einer (vor)sowjetischen Vorgeschichte von Bedeutung sind, bezieht sich das betreffende Argument nicht auf eine (schlecht gestaltete) frühe Institutionenwahl, sondern auf Pfadabhängigkeiten: Weil einerseits mit der Transformation des politischen Systems neue formale Organisationen und Institutionen etabliert worden sind, andererseits der politische Prozess weithin nach hergebrachten informellen Mustern funktioniert, sind die Verhaltensanreize für politische Unternehmer, in Parteien zu investieren, ambivalent. Zwar lagen der Duma eindeutig formale Organisationsstrukturen zugrunde, aber die Fraktionen blieben instabil, weil klientelistische Tauschgeschäfte den Fraktionswechsel förderten. Entsprechend lässt sich die Schwäche nationaler Parteien in der Peripherie damit erklären, dass die Regionaleliten ihr politisches Überleben ohne den Rückgriff auf nationale Parteien sichern konnten und daher kein Interesse daran hatten, die Autonomie ihrer Ressourcenzugänge durch die Bindung an formale, vom Zentrum aus gesteuerte Organisationen einzuschränken. ${ }^{27}$

Fasst man die Argumente zusammen, die in neoinstitutionalistischen Erklärungen angeboten werden, bleibt festzuhalten: Die Ursache für die Unterinstitutionalisierung des Parteiensystems findet sich nicht bei den Wählern, sondern bei den Eliten. Die neugeschaffenen formalen Institutionen stimulierten die Bildung von Parteien als Organisationsform des Elitenwettbewerbs um Wählerstimmen. Die ererbten institutionellen Institutionen hielten jedoch wenig Anreize für ihre Verstetigung bereit, wenn die betreffende Elitegruppe über Zugänge zu den Ressourcen des Staates verfügte, denn diese führten - anders als in Parteiendemokratien - nicht über Parteien. Eine grundsätzliche Veränderung der Lage schien Ende der 1990er Jahre nicht in Sicht.

\section{Die Institutionalisierung des Parteiensystems in der Ära Putin}

Vergleicht man Beschreibungen des russischen Parteiensystems der 1990er Jahre mit jenen im Jahre 2008 (vgl. Tabelle 2), so fallen grundsätzliche Veränderungen ins Auge: Es hat sich inzwischen mehr oder weniger voll institutionalisiert, Parteien sind als der einzige legitime Akteurstypus in der elektoralen und der parlamentarischen Arena akzeptiert. Sie sind nunmehr landesweit organisiert, und sie integrieren auch die regionalen Eliten.

Die Parteienlandschaft hat sich deutlich stabilisiert und wird nicht mehr durch beständige Neugründungen im Fluss gehalten. Unter den elf Parteien, die im Dezember 2007 zur

26 Henry E. Hale, Regime Cycles: Democracy, Autocracy, and Revolution in Post-Soviet Eurasia, in: World Politics, 58. Jg. (2005), H. 1, S. 133 - 165.

27 Grigorii V. Golosov, Electoral Systems and Party Formation in Russia: A Cross-Regional Analysis, in: Comparative Political Studies, 36. Jg. (2003), H. 8, S. 912 - 935; Mikhail Myagkov / Peter C. Ordeshook, a.a.O. (Fn. 14); Oleh Protsyk / Andrew Wilson, Centre Politics in Russia and Ukraine: Patronage, Power and Virtuality, in: Party Politics, 9. Jg. (2003), H. 6, S. 703 - 727; Kathryn Stoner-Weiss, a.a.O. (Fn. 7). 
Wahl antraten, befanden sich lediglich zwei neue Organisationen, die aber nur marginalen Zuspruch erhielten. Über 90 Prozent der Stimmen gingen an vier nationale Parteien, darunter fast zwei Drittel an „Einiges Russland“ (ER), das bereits zum dritten Mal an Parlamentswahlen teilnahm. Zusammen ein Fünftel erhielten die beiden „Dinosaurier“ des russischen Parteiensystems, die Kommunisten und die Liberal-Demokraten. Der einzige Neuling im Parlament, die Partei „Gerechtes Russland“ (GR), war im Herbst 2006 aus dem Zusammenschluss dreier Parteien entstanden, die bereits an den vorangegangenen Wahlen teilgenommen hatten.

Das auffälligste Merkmal des neuen Parteiensystems besteht in seiner „Hypofragmentierung “28. Es wird von der Patronagepartei „Einiges Russland“ in einem Maße geprägt, dass es in Sartoris Typologie an der Grenze zwischen kompetitiven und nicht-kompetitiven Systemen einzuordnen ist: Entweder handelt es sich um ein System mit einer dominanten Partei, die Wahlen noch immer prinzipiell verlieren kann, oder aber um eines, in dem eine hegemoniale Partei anderen Organisationen nur den Status von „Satelliten“ oder Juniorpartnern zubilligt. ${ }^{29}$ Nach Beendigung seiner Amtszeit als Präsident übernahm Putin, nunmehr Premierminister, im Frühjahr 2008 den Vorsitz von ER, ohne ihr formal als Mitglied beizutreten. Die Partei könnte künftig zu einer (auch formalen) Regierungspartei werden, jedoch würde es eine Umgestaltung des Regierungssystems - oder eine Veränderung der gegenwärtigen Praxis - voraussetzen, um das Präsidialkabinett in eine Parteienregierung zu transformieren.

Das politische Spektrum ist im Vergleich zu den 1990er Jahren kleiner geworden. Gegenwärtig sind 15 Parteien registriert. Neben den unten noch näher zu beschreibenden „Parteien der Macht“ - „Einiges Russland“ und „Gerechtes Russland“ - , die sich leicht rechts beziehungsweise links der Mitte des politischen Spektrums positioniert haben, handelt es sich um Organisationen, die den Familien der liberalen, (national-)kommunistischen und (rechts-)nationalistischen Parteien zugeordnet werden können. Der Wählerzuspruch zur Kommunistischen Partei hat sich auf einem erheblich niedrigeren Niveau als in den 1990er Jahren stabilisiert, der Polarisierungsgrad des Parteiensystems bildet aber nicht ab, dass sie seit dem Jahre 2003 auch die einzige verbliebene oppositionelle Kraft darstellt. Sowohl die sozial- als auch die wirtschaftsliberale Opposition („Jabloko“ und „Union der rechten Kräfte“) haben ihre parlamentarische Präsenz Ende 2007 eingebüßt und stehen vor dem organisatorischen Zerfall. Die LDPR ist zwar weiterhin in der Duma vertreten, hat sich aber in ihrem Abstimmungsverhalten bereits seit der zweiten Hälfte der 1990er Jahre meist als zuverlässiger Partner der Exekutive erwiesen. ${ }^{30}$

28 Vladimir Gelman, a.a.O. (Fn. 7), S. 547.

29 Vgl. Giovanni Sartori, a.a.O. (Fn. 10). Die aktuelle Literatur vergleicht Russland zuweilen mit Mexiko, dem Referenzfall eines hegemonialen Parteiensystems, vgl. Vladimir Gel'man, a.a.O. (Fn. 7); Regina Smyth / Anna Lowry / Brandon Wilkening, a.a.O. (Fn. 2). Vladislav Surkov, der Chefstratege in der Präsidialadministration, verweist hingegen auf das japanische Parteiensystem mit einer dominanten Partei, vgl. Vladislav Surkov, Suverenitet - eto političeskij sinonim konkurentosposobnosti (Stenogramm einer Rede in der Parteischule von ER), 7. Februar 2006 (http:// www.er.ru, Abruf am 15. September 2008).

30 In den Jahren 2000 bis 2003 beispielsweise stimmte sie für 85,6 Prozent der vom Präsidenten eingebrachten Gesetzesentwürfe. Die KPRF hingegen verhielt sich mit 27,2 Prozent Ja-Stimmen deutlich oppositionell, während die beiden liberalen Parteien „Jabloko" und „Union der Rechten Kräfte" den Gesetzesvorschlägen des Präsidenten zu 55,7 beziehungsweise 61,7 Prozent folgten, vgl. Thomas F. Remington, Presidential Support in the Russian State Duma, in: Legislative Studies Quarterly, 31. Jg. (2006), H. 1, S. 5 - 32, S. 18. 
Die Bereitschaft zur Wechselwahl ist geringer geworden, aber noch immer recht hoch. Sie hat sich jedoch qualitativ verändert. 1993 und 1995 hatten die Wähler stark auf die sich ständig neu strukturierende Parteienlandschaft reagiert, seit 1999 aber vollziehen sich die Stimmenwanderungen größtenteils zugunsten der Parteien der Macht: Unter den hochkompetitiven Bedingungen des Jahres 1999 entfielen fast 37 Prozent der Volatilität auf die beiden Rivalen „Vaterland - Ganz Russland“ und „Einheit“, womit die Wähler erstmals in großer Zahl zentristische Parteien unterstützten. ${ }^{31}$ Im Jahre 2003, als diese beiden sich bereits zu ER zusammengeschlossen hatten, profitierte neben der LDPR mit GR eine neue Patronagepartei des Kreml von diesem „Trend zur Mitte“, während die Wählerschaft von ER unverändert blieb. Die recht hohe Volatilität bei der Parlamentswahl im Dezember 2007 wiederum kam allein Putins Partei ER zugute. Sie zeigte an, dass es dieser gelungen war, sich in eine hegemoniale Position zu bringen, indem sie die Wahlen als Vorentscheidung für den im Frühjahr 2008 anstehenden Wechsel im Präsidentenamt präsentierte und dramatisierte.

Aus diesen Entwicklungen kann nun aber nicht geschlossen werden, dass in der russischen Bevölkerung das Vertrauen in die Parteien, das Parlament oder die Fairness des Wahlprozesses in den vergangenen Jahren gewachsen wäre. ${ }^{32}$ Auch liegt die durchschnittliche Wahlbeteiligung trotz nachhaltiger und manipulativer Bemühungen der Präsidialexekutive insbesondere im Jahre 2007 nicht über dem Niveau der 1990er Jahre.

Zusammenfassend lässt sich der Wandel des russischen Parteiensystems seit dem Beginn des Jahrzehnts nicht als Anzeichen einer demokratischen Konsolidierung interpretieren, sondern als „monopolistische Konsolidierung des politischen Marktes“33, mithin als eine der deutlichsten Manifestationen des allgemeinen autoritären Entwicklungstrends des politischen Systems. Die Intensität des politischen Wettbewerbs nahm erheblich ab. Bei der Parlamentswahl im Dezember 2007 erhielt ER 64,3 Prozent der Wählerstimmen. Der Präsidentschaftskandidat der beiden Parteien der Macht, Putins Wunschkandidat Sergej Medvedev, vereinte im März 2008 70,2 Prozent der Stimmen auf sich. Entsprechend einer theoretisch nicht genau begründbaren, in der Literatur aber allgemein akzeptierten Festlegung ist damit knapp der Schwellenwert überschritten, jenseits dessen politische Systeme als nicht-kompetitiv beziehungsweise „hegemonial-autoritär“ gelten. ${ }^{34}$ Die Höhe dieser beiden Wahlsiege mag zum kleineren Teil auf Manipulationen des Wahlkampfs und der Ergebnisse zurückgehen; der Schlüssel zu ihrem Verständnis liegt jedoch nicht in situativen Betrugsmanövern, sondern in der systematisch abgesicherten Dominanz der herrschenden Elitegruppe, darunter auch auf der Ebene der Wählerschaft.

\section{Gründe für die Transformation des Parteiensystems}

Gründe für die Transformation des Parteiensystems können nicht im Regierungssystem gesucht werden, denn dieses hat sich seit den 1990er Jahren nicht verändert. Auch eine

31 Regina Smyth, a.a.O. (Fn. 7).

32 Stephen White, Russians and Their Party System, in: Demokratizatsiya, 14. Jg. (2006), H. 1, S. 7 - 22, S. 9.

33 Vladimir Gelman, a.a.O. (Fn. 7), S. 550.

34 Vgl. Steven Levitsky / Lucan A. Way, a.a.O. (Fn. 2); Marc Morjé Howard / Philip G. Roessler, a.a.O. (Fn. 2). 
paradigmatische Wahlrechtsreform griff im nationalen Rahmen erstmals im Jahre 2007. Die meisten Anzeichen einer Institutionalisierung des Parteiensystems - zunehmende organisatorische Persistenz, Aufbau landesweiter Parteistrukturen, Konzentrationsprozesse im Parlament - setzten jedoch bereits Jahre zuvor ein. Folgt man der neoinstitutionalistischen Erklärungslogik, die oben skizziert wurde, sind die Ursachen dafür erstens nicht bei den Wählern zu suchen, sondern in der Sphäre des intra-elitären Wettbewerbs. Er brach am Ende von El'cins Amtszeit offen aus und wurde durch Putins Strategien der Etablierung einer Partei der Macht sowie flankierende Reformen des Parteien- und Wahlrechts wieder schrittweise befriedet. Zweitens änderte die Präsidialadministration damit die Anreizstrukturen für politische Unternehmer so, dass das Investieren in die Partei der Macht (unter Umständen auch in eine konkurrierende Partei desselben Typs) attraktiv geworden ist. Für die regierende Elitenkoalition hat dies den Vorteil, den Wettbewerb aufgrund seiner formalen Organisationsstrukturen besser regulieren und steuern zu können. Parteien, die als Basisinstitutionen der Demokratie gelten, können also auch in nicht-demokratischen politischen Systemen wichtige Funktionen der System- und Regimestabilisierung übernehmen.

\section{1. „Parteien der Macht“ im parteipolitischen Wettbewerb der 1990er Jahre}

Um die tendenziell autoritäre Transformation des russischen Parteiensystems in der Ära Putin zu verstehen, muss bei der Bedeutung von Wahlen für politische Systeme in der „Grauzone“ -,defekter Demokratien“ ebenso wie „kompetitiver Autokratien“ - angesetzt werden. Wahlen sind entscheidend für die formal-legale Legitimität der regierenden Eliten, selbst wenn deren Rekrutierung sich real über Kanäle der patronal-präsidentiellen Herrschaftsorganisation vollzieht. In diesem Zusammenhang kommt selbst unterinstitutionalisierten Parteien Bedeutung zu, und zwar zunächst als „Wahlmaschinen“, die das Elektorat kurzfristig mobilisieren.

Solche Wahlmaschinen der Exekutive waren eine charakteristische Erscheinung bereits seit den ersten freien Wahlen 1993 (vgl. Tabelle 4). Sie wurden von der Präsidialadministration jeweils wenige Monate vor einer Parlamentswahl in Gang gesetzt. In den 1990er Jahren waren sie stets kurzlebig und konstituierten keine ideologische Parteienfamilie. Ihre Aufgabe bestand darin, möglichst viele Stimmen von der kommunistischen Opposition abzuziehen, um deren Position in der Duma zu schwächen. Chancen schöpften solche Parteien aus ihrer privilegierten Situation: Als Patronageparteien der Exekutive bedienten sie sich der administrativen Ressourcen staatlicher Institutionen, finanzieller Mittel von verbündeten Großunternehmen, eines begünstigten Medienzugangs und nicht zuletzt prominenter Politiker, die als „Zugpferde“ fungierten. Nach ihrer (nur bedingt erfolgreichen) Funktionserfüllung als Wahlmaschinen wurden sie vom politischen Establishment bald wieder fallen gelassen. Die auf ihrer Grundlage gebildeten Fraktionen waren nie groß und diszipliniert genug, um die Basis einer strukturellen Mehrheit der Exekutive in der Duma abzugeben. ${ }^{35}$

35 Zoe Knox / Pete Lentini / Brad Williams, Parties of Power and Russian Politics: A Victory of the State over Civil Society, in: Problems of Post-Communism, 53. Jg. (2006), H. 1, S. 3 - 14; Regina Smyth, a.a.O. (Fn. 7). 


\begin{tabular}{|c|c|c|c|c|c|c|}
\hline \multicolumn{7}{|c|}{ Tabelle 4: "Parteien der Macht" und Dumawablen in Russland (1993 bis 2007) } \\
\hline \multirow{2}{*}{$\begin{array}{c}\text { Wahl- } \\
\text { tag }\end{array}$} & \multirow{2}{*}{$\begin{array}{c}\text { Partei } \\
\text { (Gründungsdatum) }\end{array}$} & \multirow{2}{*}{ Patron } & \multirow{2}{*}{ Parteivorsitzender } & \multicolumn{3}{|c|}{$\begin{array}{l}\text { Anteil der Mandate laut } \\
\text { Wahlergebnis in Prozent }\end{array}$} \\
\hline & & & & \begin{tabular}{|c|} 
Partei der \\
Macht
\end{tabular} & KPRF & LDPR \\
\hline $\begin{array}{l}12.12 . \\
1993\end{array}$ & $\begin{array}{l}\text { Russlands Wahl } \\
\text { (Sommer 1993) }\end{array}$ & \begin{tabular}{|l}
$\begin{array}{l}\text { Präsidial- } \\
\text { administration }\end{array}$ \\
\end{tabular} & $\begin{array}{l}\text { Egor Gajdar } \\
\text { (Vize-Premier) }\end{array}$ & 14,0 & 9,5 & 14,4 \\
\hline \multirow{2}{*}{$\begin{array}{l}17.12 . \\
1995\end{array}$} & $\begin{array}{l}\text { Unser Haus Russland } \\
\text { (Mai 1995) }\end{array}$ & \begin{tabular}{|l}
$\begin{array}{l}\text { Präsidial- } \\
\text { administration }\end{array}$ \\
\end{tabular} & $\begin{array}{l}\text { Viktor Černomyrdin } \\
\text { (Premier) }\end{array}$ & 12,2 & \multirow{2}{*}{34,9} & \multirow{2}{*}{11,3} \\
\hline & $\begin{array}{l}\text { Ivan-Rybkin-Block } \\
\text { (Juli 1995) }\end{array}$ & \begin{tabular}{|l|} 
Präsidial- \\
administration
\end{tabular} & $\begin{array}{l}\text { Ivan Rybkin (Duma-Präsi- } \\
\text { dent) }\end{array}$ & 0,7 & & \\
\hline \multirow[t]{2}{*}{$\begin{array}{l}19.12 . \\
1999\end{array}$} & $\begin{array}{l}\text { Vaterland - Ganz Russ- } \\
\text { land (Herbst } 1998 \text { / } \\
\text { formal: August 1999) }\end{array}$ & $\begin{array}{l}\text { regionale } \\
\text { Eliten }\end{array}$ & \begin{tabular}{|l} 
Jurij Lužkov (OB \\
Moskau), Evgenij \\
Primakov (ehemaliger \\
Premier) \\
\end{tabular} & 15,0 & \multirow[t]{2}{*}{25,8} & \multirow[t]{2}{*}{3,9} \\
\hline & \begin{tabular}{|l|} 
Einheit („Bär") \\
(September 1999)
\end{tabular} & \begin{tabular}{|l|} 
Präsidial- \\
administration
\end{tabular} & $\begin{array}{l}\text { Sergej Šojgu (Minister, } \\
\text { „Putin-Lager“) }\end{array}$ & 16,6 & & \\
\hline \multirow[b]{2}{*}{$\begin{array}{l}7.12 . \\
2003\end{array}$} & $\begin{array}{l}\text { Einiges Russland (April } \\
\text { bis Dezember 2001) }\end{array}$ & \begin{tabular}{|l|} 
Präsidial- \\
administration
\end{tabular} & $\begin{array}{l}\text { Boris Gryzlov (Duma- } \\
\text { Vorsitzender) }\end{array}$ & 49,3 & \multirow[b]{2}{*}{11,6} & \multirow[b]{2}{*}{8,0} \\
\hline & \begin{tabular}{|l} 
Heimat \\
(September 2003)
\end{tabular} & $\begin{array}{l}\text { Präsidial- } \\
\text { administration }\end{array}$ & $\begin{array}{l}\text { Sergej Glaz'ev, Dmitrij } \\
\text { Rogozin (Duma-Abgeord- } \\
\text { nete, bekannte Politiker) }\end{array}$ & 8,2 & & \\
\hline \multirow{2}{*}{$\begin{array}{l}2.12 . \\
2007\end{array}$} & Einiges Russland & \begin{tabular}{|l|} 
Präsidial- \\
administration
\end{tabular} & $\begin{array}{l}\text { Boris Gryzlov (Duma- } \\
\text { Vorsitzender) }\end{array}$ & 69,9 & \multirow{2}{*}{12,7} & \multirow{2}{*}{8,9} \\
\hline & \begin{tabular}{|l|}
$\begin{array}{l}\text { Gerechtes Russland } \\
\text { (Oktober 2006) }\end{array}$ \\
\end{tabular} & $\begin{array}{l}\text { Präsidial- } \\
\text { administration }\end{array}$ & $\begin{array}{l}\text { Sergej Mironov (Vorsitzen- } \\
\text { der des Föderationsrates) }\end{array}$ & 8,4 & & \\
\hline
\end{tabular}

Die Rolle von Parteien der Macht änderte sich gegen Ende der Amtszeit El'cins. Da sich die politischen Eliten über die künftige Besetzung des Präsidentenamts (anders als acht Jahre später) intern nicht einigen konnten, brach offene Konkurrenz zwischen ihren Fraktionen aus, die sich auch in der elektoralen Arena manifestierte. ${ }^{36}$ Aufgrund des russischen Wahlkalenders bedeutete die Parlamentswahl eine Vorentscheidung für die drei Monate später stattfindende Präsidentschaftswahl. Die Parteienbildung erhielt davon starke Impulse.

Während die jeweils zwei Parteien der Macht, die 1995, 2003 und 2007 zur Wahl antraten, Bemühungen der Präsidialadministration geschuldet waren, ein Zweiparteiensystem zu lancieren beziehungsweise als „Spoiler“ gegenüber den Kommunisten zu wirken ${ }^{37}$, repräsentierten sie im Jahre 1999 tatsächlich zwei rivalisierende Elitenbündnisse im Kampf um die El'cin-Nachfolge: „Vaterland - Ganz Russland“ stellte eine eher lose Koalition regionaler Elitennetzwerke unter Führung des ehemaligen Premierministers Jewgeni Primakov und des Moskauer Oberbürgermeisters Jurij Lužkov dar, während „Einheit“ die gezielt aus der

36 Dies ist eine typische Situation, wenn Regime des patronalen Präsidentialismus vor dem Problem stehen, einen Nachfolger für den bisherigen Amtsinhaber zu rekrutieren. Sie stellt ihre Reproduktionsfähigkeit auf die Probe, vgl. Henry E. Hale, a.a.O. (Fn. 26).

37 Michael McFaul, a.a.O. (Fn. 22); Zoe Knox / Pete Lentini / Brad Williams, a.a.O. (Fn. 35). 
Präsidialadministration gesteuerte Gegengründung einer durch El'cin protegierten Gruppe um den neuen Premier Putin verkörperte. Sie positionierten sich programmatisch diffus als links- beziehungsweise rechtszentristische „Projektparteien“"38. Dies verschaffte ihnen zu diesem Zeitpunkt Wettbewerbsvorteile gegenüber der stärker profilierten kommunistischen und liberalen Opposition, denn in der Wählerschaft hatten sich gegen Ende der 1990er Jahre Tendenzen der De-Ideologisierung ausgebreitet, und die Polarisierung des Parteiensystems war geringer geworden (siehe Abschnitt 2.1.). ${ }^{39}$

Die Situation entwickelte sich dramatisch und wurde eher überraschend, aber nur knapp zugunsten der Partei Putins entschieden, und zwar in erster Linie, weil aufgrund der geschickten Strategie von „Einheit“ große Teile der Regionaleliten mit ihrer Wählerschaft zu ihr umschwenkten. ${ }^{40}$ Der intra-elitäre Wettbewerb war endgültig beigelegt, als El'cin durch seinen vorzeitigen Rücktritt zum Jahreswechsel 1999/2000 seinem Protegé den Zugang zur Schlüsselposition im Staat eröffnete. Der neue Präsident lernte aus dieser Erfahrung und transformierte „seine“ Partei der Macht in ein Instrument der intra-elitären Konsensbildung.

\subsection{Institutionalisierung der „Partei der Macht“ als Wahl-, Parlaments- und Mitgliederpartei}

„Einiges Russland“, 2001 aus dem Zusammenschluss der Fraktionen „Einheit“ und „Vaterland - Ganz Russland“ entstanden, ist die erste Partei der Macht, die im russischen Parteiensystem dauerhaft überleben konnte. ${ }^{41}$ Dies liegt daran, dass sich das Verhältnis der Präsidialexekutive gegenüber diesem Organisationstypus nach der Jahrtausendwende grundsätzlich änderte. Das Ziel, eine lediglich kurzlebige Wahlmaschine zu schaffen, wurde durch die Strategie der Institutionalisierung einer „Partei des Präsidenten“ ersetzt. Mit ER wurde zunächst eine zuverlässige parlamentarische Partei etabliert, deren Aktivitäten durch die Präsidialadministration gesteuert wurden. Sie bewährte sich bei den Duma-Wahlen 2003 und 2007 als Wählerpartei und konstituierte sich gegen Ende der Amtszeit Putins auch als Mitgliederpartei.

„Einheit“ und „Vaterland“ waren - entsprechend der neopatrimonialen Logik des Elitenwettbewerbs in personal-präsidentiellen Systemen - klientelistische Personenbündnisse ohne ernstzunehmende ideologisch-programmatische Differenzen gewesen. Jede von ihnen hatte im Jahre 1999 Wählerzuspruch erhalten, der über den für frühere Parteien der Macht

38 Richard Sakwa, The 2003-2004 Russian Elections and Prospects for Democracy, in: Europe-Asia Studies, 57. Jg. (2005), H. 3, S. $369-398$.

39 Regina Smyth, a.a.O. (Fn. 7).

40 Timothy J. Colton / Michael McFaul, Reinventing Russia’s Party of Power: „Unity” and the 1999 Duma Election, in: Post-Soviet Affairs, 16. Jg. (2000), H. 3, S. 201 - 224; Vladimir Gelman, a.a.O. (Fn. 7), S. 552 - 554; Henry E. Hale, The Origins of United Russia and the Putin Presidency: The Role of Contingency in Party-System Development, in: Demokratizatsiya, 12. Jg. (2004), H. 2, S. 169 - 194; Zoe Knox / Pete Lentini / Brad Williams, a.a.O. (Fn. 35); Mikhail Myagkov / Peter C. Ordeshook, a.a.O. (Fn. 14).

41 Regina Smyth, a.a.O. (Fn. 7); Regina Smyth / Anna Lowry / Brandon Wilkening, a.a.O. (Fn. 2); Vladimir Gelman, Party Politics in Russia: From Competition to Hierarchy, in: Europe-Asia Studies, 60. Jg. (2008), H. 6, S. 913 - 930, S. 920 ff. 
hinausging. Der Rückzug des Führungspersonals in der Verlierergruppe („Vaterland“) machte den Weg frei, um eine stabile parlamentarische Mehrheit in der Duma zu schmieden. Durch klientelistische Tauschgeschäfte, wie sie auch zuvor üblich gewesen waren, wurden wiederum erhebliche Umstrukturierungen innerhalb des Parlaments angeregt: Waren im Dezember 1999 nur 139 Kandidaten der beiden Parteien der Macht in das Parlament eingezogen, konstituierte sich die Duma einen Monat später bereits mit 222 Abgeordneten in vier pro-präsidentiellen Fraktionen beziehungsweise Gruppen; ihre Zahl erhöhte sich bis zum Frühjahr 2001 auf 235, was die absolute Stimmenmehrheit im Parlament sicherte; die Parlamentsfraktionen „Einheit“ und „Vaterland“ schlossen sich 2001 auch formal zusammen (vgl. Tabelle 3, Spalte 2000-2003 ${ }^{42}$ ).

Neu gegenüber der Praxis der El'cin-Ära ${ }^{43}$ war, dass die Kohäsion der präsidialen Unterstützerkoalition nun nicht mehr ad hoc, sondern mittels formal-institutioneller Anreize gesichert wurde. Die bedeutendsten Innovationen waren die Schaffung eines Koordinationsgremiums, im Rahmen dessen die pro-präsidentiellen Parlamentsfraktionen ihr Abstimmungsverhalten vorab regelten, sowie die Einrichtung von „Null-Lesungen“, während derer die Gesetzesentwürfe der Exekutive beraten wurden, bevor sie in die erste offizielle Lesung der Duma gingen. ${ }^{44}$ Im Ergebnis entstand so erstmals im postkommunistischen Russland eine dominante, auch formal institutionalisierte parlamentarische Partei der Macht. Ihre systematische Unterstützung des Präsidenten war effizienter und weitaus leichter zu koordinieren als mittels der klientelistischen Aushandlungen in den vorangegangenen Parlamenten.

Die Dynamik des Laakso-Taagepera-Indexes (vgl. Tabelle 2, zweite und dritte Spalte) bildet die zunehmende Konzentration des Parteiensystems ab. Wie deutlich zu erkennen ist, fand der qualitative Durchbruch nicht auf der Ebene der Wähler und aufgrund ihres Votums statt, sondern durch Veränderungen im Parlament - durch den Fraktionsbeitritt von Abgeordneten kurz nach der Wahl 1999, durch den Zusammenschluss der beiden propräsidentiellen Fraktionen im Frühjahr 2001 und bei der Konstituierung der Duma im Januar 2004: Alle 32 Abgeordneten kleiner Parteien und 50 der 68 unabhängigen Mandatsträger hatten sich über den Jahreswechsel entschieden, der Fraktion „Einiges Russland“ beizutreten. ${ }^{45}$ Die Wanderungsbewegungen in den vorangegangenen Wahlperioden waren nie derart gleichgerichtet gewesen.

Die Institutionalisierung von ER in ihrer Dimension als Mitgliederpartei begann mit der Rekrutierung diverser staatlicher und administrativer Elitegruppen, insbesondere in den Regionen. 2006 gehörten ihr bereits mehr als zwei Drittel der 88 Gouverneure und 107 der

42 In den Angaben zum Ende der Wahlperiode wurden alle vier pro-präsidentiellen Fraktionen zu einer Fraktion zusammengefasst, obwohl die aus unabhängigen Mandatsträgern gebildeten Gruppen „Abgeordnete des Volkes“ und „Russische Regionen“ formal selbständig blieben.

43 Vgl. dazu Eugene Huskey, Democracy and Institutional Design in Russia, in: Demokratizatsiya, 4. Jg. (1996), H. 4, S. 453 - 473; Thomas F. Remington, a.a.O. (Fn. 30).

44 Regina Smyth, a.a.O. (Fn. 7); Thomas F. Remington, a.a.O. (Fn. 30). Die Abstimmungsdisziplin der unabhängigen Abgeordneten blieb nichtsdestotrotz weniger diszipliniert als die der Fraktion „Einiges Russland“.

45 Unabhängigen Mandatsträgern sollen für den Wechsel einmalig 50.000 US-Dollar sowie monatliche Zahlungen von 5.000 Dollar angeboten worden sein, vgl. Igor' Dmitriev, Duma - for sale. Kto i skol'ko platit za deputatskoe lobbi, in: Versija vom 9. November 2004. 
178 Mitglieder der Zweiten Parlamentskammer sowie einige föderale Minister an. Bei den Duma-Wahlen Ende 2007 kandidierten 65 der 85 Regionaloberhäupter auf den Listen der Partei. In Vorbereitung zu den Wahlen 2007 unternahm sie verstärkte Bemühungen, Mitglieder und Sympathisanten zu werben und die Beziehungen zu ihnen zu institutionalisieren. Im Mai 2008 zählte die Partei bereits mehr als zwei Millionen Mitglieder und damit mehr als zehnmal so viel wie die KPRF.

Die Mobilisierungsstrategien von ER sind vielfältig. Mit gesellschaftlichen Organisationen werden auf nationaler und regionaler Ebene Kooperationsverträge vereinbart. Die Zahl dieser Partner, deren Spektrum vom Imkerverein bis zu Gewerkschaften und Unternehmerverbänden reicht, ist zwischen Frühjahr 2006 und Herbst 2008 von 30 auf über 70 gestiegen. Für die Mobilisierung individueller Bürger wurden „Sympathisantenräte“ in allen russischen Regionen gegründet, außerdem entstanden bis zum Herbst 2008 über 2000 solcher Räte auf der lokalen Ebene. ${ }^{46}$ Auch der Einsatz von staatlichen Ressourcen im nominellen Rahmen anderer Reformprojekte, insbesondere der Ausbau des Verwaltungsapparates und die Gehaltserhöhungen für die Staatsbediensteten, die 60 Prozent der Mitglieder stellen, sowie die Ausrufung der vier großen „Nationalen Projekte“ in den Bereichen Gesundheit, Bildung, Wohnen und Landwirtschaft sind als Instrumente der Mobilisierung von Mitgliedern und Wählern interpretiert worden; die Grenzen zwischen den Vorhaben des Staates und der Partei sind also fließend. ${ }^{47}$

\subsection{Die Einschränkung des Wettbewerbs durch institutionelle Reformen}

„Einiges Russland“ sicherte die reibungslose Verabschiedung von Putins ambitioniertem Programm institutioneller Reformen in der Duma. Dazu gehörten auch Gesetzesprojekte, die auf die Reduzierung des parteipolitischen Wettbewerbs zielten, indem sie hohe Eintrittsschranken zum politischen Markt festlegten. Kleine, regionale oder organisatorisch instabile Organisationen wurden auf formal-legalem Weg vom Kampf um Wählerstimmen ausgeschlossen. Das Spektrum organisierbarer Interessen wurde gesetzlich eingeschränkt: Das neue Parteiengesetz (verabschiedet 2001, modifiziert 2004) legte hohe Maßstäbe an die Zulassung einer Partei an. Sie muss nunmehr mindestens 50.000 Mitglieder nachweisen und in mindestens zwei Dritteln aller Regionen aktiv sein. Das Recht, an Wahlen teilzunehmen, wurde exklusiv auf Parteien beschränkt. ${ }^{48}$ Im Gegenzug zur Limitierung der privaten Finanzierung von Parteien erfolgte die Stärkung der staatlichen Parteienfinanzierung. Um eine sozialstrukturelle Unterfütterung des Parteienwettbewerbs zu verhindern - was für die Parteiensysteme Westeuropas historisch zwar konstitutiv gewesen ist, in Russland aber als Gefahr der Polarisierung und Radikalisierung interpretiert wurde -, untersagt das Gesetz, sich programmatisch auf soziale Gruppen, Religionszugehörigkeit, Geschlecht, Beruf oder Herkunft zu berufen.

Das zweite wichtige Gesetz betraf das Wahlrecht. Es war seit 1993 nicht verändert worden, womit es - neben dem estnischen und dem turkmenischen Wahlrecht - eine Aus-

46 Eigenangaben der Partei (http://www.er.ru, Abrufe am 25. Mai 2006 und 22. September 2008).

47 Regina Smyth / Anna Lowry / Brandon Wilkening, a.a.O. (Fn. 2), S. 126.

48 In den 1990er Jahren waren auch ,gesellschaftliche Organisationen“, „Bewegungen“ und „Wahlvereinigungen" zur Teilnahme an Wahlen zugelassen. 
nahme unter den postkommunistischen Ländern darstellte. ${ }^{49}$ Das 2005 verabschiedete Wahlgesetz schaffte das Majorz-Segment ab und installierte ein Verhältniswahlrecht nach Parteilisten mit einer Sperrklausel von sieben Prozent. ${ }^{50}$ Bei seiner ersten landesweiten Anwendung im Dezember 2007 kam es nicht zu signifikanten Veränderungen im Parlament, was die Zahl und das Kräfteverhältnis zwischen „Einiges Russland“, den Kommunisten und Liberal-Demokraten betraf. Neu und legitimitätssteigernd war vielmehr, dass dieses Ergebnis unmittelbar und transparent aus der Übertragung von Wählerstimmen in Mandate also direkt aus dem ausgezählten Wählerwillen - erwuchs.

Der Zweck dieser Reformen bestand darin, den politischen Wettbewerb auf eine überschaubare Teilnehmerzahl zu reduzieren und die Rolle von Organisationen (und ihren Führungen) gegenüber Einzelpersonen zu stärken, was insbesondere das eigensinnige Wahl- und Abstimmungsverhalten von Regionalpolitikern unterbinden sollte. In diesem Sinne sind die Neuregelungen des Parteien-, Abgeordneten ${ }^{51}$ - und Wahlrechts in einem inhaltlichen $\mathrm{Zu}$ sammenhang mit den Föderalismusreformen zu sehen: Die regionalen Eliten wurden in landesweit agierende Parteien eingebunden; in der Duma gibt es keine unabhängigen Mandatsträger mehr. Dem liegt dasselbe Bestreben zugrunde, die regionalen Eliten vom Zentrum der Macht her zu kontrollieren wie dem größten Teil der Föderalismusreformen, darunter der Etablierung der Föderalbezirke, der Abschaffung der Gouverneurswahlen und der Einrichtung des konsultativen Staatsrats als Ersatz dafür, dass die Regionaloberhäupter seit dem Jahr 2000 nicht mehr in der Zweiten Parlamentskammer vertreten sind. ${ }^{52}$

\section{Institutionalisiertes Parteiensystem und erfolgreiche Regimereproduktion}

Resümiert man die Ursachen für den Wandel des russischen Parteiensystems, so ist festzuhalten: Seine aktuelle Schließung geht nicht primär auf die neue Parteien- und Wahlgesetzgebung zurück. Diese sichert vielmehr die bereits in den Jahren zuvor durchgesetzte Dominanz von „Einiges Russland“ rechtlich ab und wurde ihrerseits erst aufgrund dieser Dominanz möglich. Das neue Parteien- und Wahlrecht erzielte seine Wirkungen nur deshalb, weil sich die Patronage der Präsidialadministration für ER als stabil erwies und es auch im Wahlkampf 2007 gelang, die Mehrheit der Wählerstimmen für sie zu mobilisieren.

49 Jack Bielasiak, Regime Diversity and Electoral Systems in Post-Communism, in: Journal of Communist Studies \& Transition Politics, 22. Jg. (2006), H. 4, S. $407-430$.

50 Die Sperrklausel gilt nur, wenn sie von mindestens zwei Parteien überwunden wird, die zusammen mehr als 60 Prozent der Wählerstimmen auf sich vereinen. Falls dies nur einer Partei gelingt, erhält auch die zweitstärkste Partei entsprechend ihres Stimmenanteils Sitze im Parlament. Vgl. dazu auch Bernd Wieser, Das Parteienaufösungsurteil des russischen Verfassungsgerichts vom 16. Juli 2007, in: Osteuropa Recht, 54. Jg. (2008), H. 1-2, S. 2 - 17. Details auch bei Angelika Nußberger / Dmitry Marenkov, Wahlgesetz als Steuerungsmechanismus: Zu den neuen rechtlichen Grundlagen der Duma-Wahlen im Dezember 2007, in: Russlandanalysen, Nr. 146, 26. Oktober 2007, S. $2-5$.

51 Entsprechend der revidierten Abgeordnetenverordnung vom Oktober 2007 zieht der Austritt aus einer Fraktion den Verlust des Mandates nach sich (vgl. Reglement der Staatsduma, Verordnung vom 22. Januar 1998, Nr. 2134-IIGD, revidiert am 9. Oktober 2007, Nr. 5134-4GD, Art. 18 (http://www.duma.gov.ru/, Abruf am 5. November 2008).

52 Vgl. auch Thomas F. Remington, a.a.O. (Fn. 30), Vladimir Gel'man, a.a.O. (Fn. 7), S. 552. 


\subsection{Systemreformen nach einem autoritären Plan?}

Die Institutionalisierung von „Einiges Russland“ als elektorale, parlamentarische und Mitgliederpartei ist demnach der Schlüssel, um den Wandel des russischen Parteiensystems wie auch die Reproduktion des unter Putin konsolidierten Regimes über seine Amtszeit hinaus zu verstehen. Mittels dieser Partei ist es gelungen, ambitionierte Politiker aus unterschiedlichen Elitenfraktionen, insbesondere die regionalen Eliten, zu integrieren und die Unterstützung der Wählerschaft zu sichern. Sie hat sich bei der Übergabe des Präsidentenamtes von Putin auf Medvedev bewährt, denn sie erwies sich wiederum als hocheffiziente Wahlmaschine, und zwar sogar beim „Verleih“ an einen lizenzierten Kandidaten. Ob dies auf eine fortschreitende „Ent-Persönlichung“ des Regimes beziehungsweise die „Transsubstantation" von Putins Reputation und Führungsqualitäten von seiner Person auf ER ${ }^{3}$ hinweist, wird sich erst im Rückblick weiterer Entwicklungen zeigen. Sicher ist hingegen, dass es mit Hilfe der Partei offenbar gelang, anders als 1999 den intra-elitären Wettbewerb um die Nachfolge des Präsidenten innerhalb des „Machtblocks“ beizulegen und nicht auf die Wählerebene durchschlagen zu lassen.

Damit speist sich der allgemeine Befund der (autoritären) Konsolidierung und Institutionalisierung des russischen Parteiensystems fast ausschließlich daraus, dass es dem präsidialen Zweig der Exekutive gelungen ist, eine elektoral und parlamentarisch stabile, formal institutionalisierte Gefolgschaft in Gestalt von „Einiges Russland“ zu gründen und ihr Dominanz zu verschaffen. Die Veränderungen bei den anderen relevanten Parteien des russischen Parteiensystems sind weit weniger auffällig, sieht man davon ab, dass die Parteienund Wahlrechtsreformen offenbar starke Impulse für die binnenorganisatorische Entwicklung der lebensfähigeren Organisationen der Opposition gegeben haben und die Zahl der registrierten Parteien aufgrund von anspruchsvollen Rechtsvorschriften zurückgegangen ist. Alles zusammen hat zur Folge, dass sich das Parteiensystem von einem hochfragmentierten, zentrifugalen Wettbewerbssystem mit schwachen Akteuren zu einem konzentrierten, zentripetalen System mit einem dominanten beziehungsweise hegemonialen Akteur in der Rolle einer „Staatspartei“ ${ }^{4} 4$ gewandelt hat.

Diese Entwicklung ist exemplarisch für den Unterschied zwischen der Putin- und der El'cin-Ära. Der amorphe und fluide politische Raum der 1990er Jahre ist zunehmend strukturiert worden, weil es der Präsidialadministration gelang, die Organisationsprozesse nichtstaatlicher kollektiver Akteure gezielt und nachhaltig zu beeinflussen. Ihre Absicht bestand darin, grundlegende politische Prozesse aus einem handlungsfähigen Machtzentrum heraus effizient zu steuern. Anders als El'cin setzte Putin darauf, die formale Institutionalisierung des politischen Regimes voranzutreiben. Die Parteien, durch die die regierende Elitengruppe bei Wahlen in den 1990er Jahren repräsentiert wurde, blieben stets „Projektparteien“, die als Wahlmaschinen eingesetzt wurden, sich aber danach weder auf Wählerebene noch als Mitgliederparteien stabilisierten. „Einiges Russland“ hat sich hingegen längerfristig als Akteur in allen drei Dimensionen institutionalisiert. Das ermöglichte zunächst, konkurrierende Elitengruppen zu integrieren und einen reibungslosen Machtübergang im Amt des

53 Regina Smyth / Anna Lowry / Brandon Wilkening, a.a.O. (Fn. 2), S. 124.

54 Vladimir Gel'man, a.a.O. (Fn. 41), S. 922, in Absetzung vom „Parteistaat“ der Sowjetzeit; so auch Regina Smyth, a.a.O. (Fn. 7), S. 563. 
Präsidenten zu sichern. Die Strategie der formalen Institutionalisierung erwies sich damit als höchst erfolgreich. Sie setzte eine sich selbst verstärkende Dynamik in Gang, die einerseits die ohnehin schwache Opposition noch weiter schwächte, andererseits die politische und Regimestabilität deutlich verbesserte.

Das institutionelle Umbauprojekt des politischen Systems während der Amtszeit Putins wird von externen Beobachtern oft als umstandslose Umsetzung eines auf dem Reißbrett ersonnenen „autoritären Plans“ interpretiert. Anhand der Entwicklungen des Parteiensystems ist aber zu erkennen, dass dies eine unzulässige Vereinfachung ist. Die Prämisse der „politischen Ingenieure" um Putin bestand darin, dass gesellschaftliche Stabilität und Wachstum nicht durch Selbstorganisation und das „freie Spiel“ pluralistischer Kräfte zu sichern seien, sondern nur durch den Rückgriff auf hierarchische Steuerung „von oben“, aus dem Zentrum der staatlichen Exekutive heraus. Diese im normativen Sinne autoritäre Überzeugung spiegelte sich wider in einer Politik institutioneller Reformen, die auf den Ausbau der „Machtvertikale“ und ausgeprägte staatliche Interventionen in Gesellschaft und Wirtschaft zielte. Dass diese Politik erfolgreich war, ist aber in erster Linie auf den lernfähigen Umgang mit sich verändernden Situationen zurückzuführen. Zu beobachten war dabei ein Zusammenwirken ambitionierter Steuerungsbemühungen „,von oben“ mit der Schwäche der Steuerungsadressaten in Politik und Gesellschaft. Ausgehend von der Logik des Machterhalts hat die Präsidialadministration auf die sich bietenden Anlässe und Gelegenheiten strategisch klug mit Versuchen der institutionellen Absicherung ihrer Positionen reagiert sowie Informations- und Handlungsdefizite anderer Akteure genutzt und verstärkt. Dabei ließ sie sich durchaus auch von dem Ziel einer innen- wie außenpolitischen, ökonomischen und sozialen „Wiedergeburt Russlands" leiten, das über rein egoistische Herrschaftsinteressen hinausging und kompatibel mit diesen verfolgt wurde.

Die Ergebnisse des institutionellen Wandels in der Putin-Ära werden ebenfalls regelmäßig als Re-Autoritarisierung interpretiert. Wie sich aber besonders anhand der Wahlrechtsreform zeigt, ist eine korrekte Bewertung weitaus komplizierter: Die Einführung des Verhältniswahlrechts wird von anerkannten Experten für junge Demokratien üblicherweise empfohlen ${ }^{55}$, und empirisch lässt sich nachweisen, dass sie in den 1990er Jahren insbesondere in jenen Ländern Ostmitteleuropas vollzogen wurde, deren Demokratiequalität vergleichsweise hoch war. ${ }^{56}$ In Russland hingegen zementierte das Verhältniswahlrecht bei seiner ersten landesweiten Anwendung zu den Duma-Wahlen im Dezember 2007 die Hegemonie der präsidentiellen Patronagepartei. Ebenso wie die meisten anderen formal-institutionellen Reformen Putins war seine Einführung gewissermaßen neutral. Es entfaltete seine autoritäre Wirkung erst im Kontext informeller Institutionen des "Superpräsidentialismus" und der autoritären politischen Dynamik des gesamten Systems. Das russische Beispiel illustriert daher ein weiteres Mal die Erkenntnis der vergleichenden Wahlsystemforschung, dass ähnliche Institutionen in unterschiedlichen Kontexten sehr unterschiedliche Wirkungen entfalten können und dass „electoral engineering" tatsächlich ein mächtiges Instrument der politischen Eliten sein kann - eventuell allerdings nicht, um eine bestimmte Struktur des Parteiensystems hervorzubringen, sondern vielmehr, um sie nachhaltig abzusichern.

55 Vgl. Arend Lijphart, Constitutional Design for Divided Societies, in: Journal of Democracy, 15. Jg. (2004), H. 2, S. $96-109$.

56 Jack Bielasiak, a.a.O.(Fn. 49). 
Mehr als eine Fußnote wert ist, dass die Reformdesigner politikwissenschaftlich wohlinformiert sind. Es finden sich systematische Verweise auf Erfahrungen westlicher Demokratien und Befunde der vergleichenden Forschung. So bezieht sich beispielsweise Vladislav Surkov, der Chefstratege in der Präsidialadministration, auf Samuel Huntington, um die normative Überlegenheit von Zweiparteiensystemen beziehungsweise Parteiensystemen mit einer dominanten Partei zu begründen. ${ }^{57}$ Von El'cin wurde dieses Projekt übrigens schon Mitte der 1990 er Jahre offensiv vertreten, der unter Berufung auf Maurice Duvergers „soziologische Gesetze“ allerdings die Einführung des Mehrheitswahlrechts für geboten hielt. ${ }^{58}$

\subsection{Ausblick}

Das aktuelle Wahlgesetz, die Willenserklärungen der Reformdesigner ${ }^{59}$ und nicht zuletzt die Patronage für die zweite, bisher wenig bedeutende Partei der Macht („Gerechtes Russland“) zielen nicht auf ein Einparteisystem, sondern lassen erkennen, dass Spielräume für eine Opposition vorgesehen sind. Die Frage ist aber, welche Parteien sie dauerhaft nutzen können. Neben den Kommunisten und den Liberal-Demokraten eröffnet sich eine Chance für eine zweite präsidententreue Partei und damit für einen stark zentripetalen Wettbewerb, bei dem es um die Wahl zwischen nur graduell unterscheidbaren Alternativen geht. Wie groß diese Chance ist und inwiefern dabei klare politisch-programmatische Profile entwickelt werden können, lässt sich gegenwärtig nicht bestimmen.

Die (insbesondere liberale) Opposition hat in der Putin-Ära insgesamt an politischer Bedeutung verloren. ${ }^{60}$ Dies ist zu einem großen Teil in den Institutionalisierungsstrategien der Präsidialadministration begründet, die „Einiges Russland“ eine hegemoniale Position verschafft haben; es liegt jedoch auch an weiteren Ursachen, wie etwa Asymmetrien im Zugang zu den Massenmedien und administrativen Steuerungsmethoden gegenüber nichtstaatlichen Akteuren. Darüber hinaus wirken die geringe Nachfrage der Wähler insbesondere nach „westlich-liberalen“ Gesellschaftsprojekten, die durch Erfahrungen der 1990er Jahre gespeist werden, sowie endogene Probleme der Opposition, die von elitären Strategien der Wählermobilisierung bis hin zu internen Personalquerelen und ungeeigneten Bündnisstrategien reichen. ${ }^{61}$

57 Vladislav Surkov, Suverenitet - političeskij sinonim konkurentosposobnosti (2006), in: ders., Teksty 97-07, Moskva 2008, S. 125 - 173, S. 149. Nicht zuletzt bezieht sich das gesamte Konzept der „souveränen Demokratie“ auf die Verarbeitung von Erkenntnissen der europäischen politischen Theorie, darunter etwa Carl Schmitts, und der Erfahrungen westlicher Demokratien, woraus die Notwendigkeit abgeleitet wird, in Ländern der späten Demokratisierung kontextangepasste Institutionensysteme zu installieren. Vgl. auch Ivan Krastev, Rossija kak „drugaja Evropa”, in: Rossija v global'noj politike, 19. August 2007, H. 4 (http://www.globalaffairs.ru/numbers/27/ 8065.html, Abruf am 24. Juni 2008).

58 Michael McFaul, a.a.O. (Fn. 22), S. 42.

59 Zum Beispiel Vladislav Surkov auf einer Parteikonferenz von „Einiges Russland“ am 30. Mai 2008, offizielle Homepage von Gerechtes Russland (http://www.spravedlivo.ru/news/6335.smx, Abruf am 11. Juli 2008).

60 Zur Charakteristik der Oppositionsparteien Vladimir Gelman, a.a.O. (Fn. 41), S. 922 - 927.

61 Analysen der Schwäche oppositioneller Parteien bei Vladimir Gelman, Political Opposition in Russia, in: Russian Politics and Law, 43. Jg. (2005), H. 3, S. 25 - 50; Henry E. Hale, Yabloko and the Challenge of Building a Liberal Party in Russia, in: Europe-Asia Studies, 56. Jg. (2004), H. 7 , 
Putins Reformprojekt hat durch die Institutionalisierung formaler Spielregeln Veränderungen im neopatrimonialen, patronal-präsidentiellen Typ der Herrschaftsorganisation vorangetrieben. Substantiell handelt es sich dabei um Anzeichen der bürokratischen Rationalisierung, Modernisierung und auch Verrechtlichung von politischen Praktiken. So fördert die Privilegierung von Parteien, wie sie in der Parteien- und Wahlgesetzgebung der letzten Jahre zum Ausdruck kommt, tatsächlich die Institutionalisierung des Parteiensystems, indem sie Anreize für politische Unternehmer schafft, in lebensfähige formale politische Organisationen zu investieren.

Die damit eingeleitete Entwicklung mag zu einem „partei-basierten Autoritarismus“62 führen, dessen Regime auf einer stärker formalisierten Grundlage beruht als der gegenwärtige „patronale Präsidentialismus“. Sie könnte dauerhaft regimestabilisierend wirken, weil mit der hegemonialen Partei ein Instrument geschaffen wurde, den für den Regimeerhalt wichtigen Elitenkonsens zu sichern. Die Lösung der Nachfolgefrage für das politische Spitzenamt - der verwundbarste Punkt im Lebenszyklus personalistischer Regime - müsste dann auch beim nächsten Mal nicht in eine Nachfolgekrise münden. Zunächst aber sollte aufmerksam beobachtet werden, wie sich die Beziehungen zwischen dem präsidialen Strang der Exekutive und der Regierung gestalten. Nicht ausgeschlossen ist, dass „Einiges Russland“, nunmehr als „Partei des Premierministers“, sich (neben Putin selbst) als eine der Ressourcen erweist, mit der die Regierung - in den vergangenen Jahren faktisch ein reines Präsidialkabinett - ihr Gewicht im politischen Entscheidungssystem erhöhen kann.

Dass über die formale Institutionalisierung und Integration von Loyalitätsnetzwerken in Form von Parteien auch ein Weg zu einem inhaltlich bedeutsamen Wettbewerb politischprogrammatischer Alternativen eröffnet werden könnte, ist mittelfristig nicht undenkbar. Allerdings setzt dies mindestens voraus, dass die Institutionalisierungsprozesse der vergangenen Jahre mehr sind als lediglich die Formalisierung informell-klientelistischer Strukturen der politischen Interaktionen, also sich nicht auf eine „personalisierte Institutionalisierung" beschränken, mit der sich der zentrale Akteur des politischen Systems, Putin (und seine Umgebung), gesichert hat, auch nach seinem Ausscheiden aus der Schlüsselinstitution des politischen Systems, dem Präsidentenamt, einen formalen Zugang zu politischen Ressourcen zu besitzen. $\mathrm{Ob}$ dies der Fall ist, lässt sich gegenwärtig nicht beurteilen. Mehr noch: Es dürfte auch in der Realität noch nicht feststehen.

62 Vladimir Gel'man, a.a.O. (Fn. 41). 